\title{
Iterative Learning Identification for Single Image Information Hiding
}

\author{
Ying Kong ${ }^{1}$ and Hui-Juan $\mathrm{Lu}^{2}$ \\ ${ }^{1}$ School of Information and Electronic Engineering, Zhejiang University of \\ Science and Technology, Hangzhou, China \\ kongying-888@163.com \\ ${ }^{2}$ College of Information Engineering, China Jiliang University, Hangzhou, China \\ hjlu@cjlu.edu.cn
}

\begin{abstract}
This paper presents a digital image information hiding scheme based on chaotic mapping and multi-blending, and the recovery of the hidden information is carried out from iterative learning identification perspective. With the proposed scheme the digital image information is added to a parameter of the chaotic system undertaken. An iterative learning identification algorithm is proposed for the recovering purpose. Experimental results demonstrate that the digital image watermarking can be recovered completely, and the learning algorithm is able to resist various types of attacks.
\end{abstract}

Keywords: iterative learning identification, information hiding, chaotic mapping, multi-blending

\section{Introduction}

With the development of the Internet and digital communication technologies, digital image as one of the most important information carriers, has been widely transmitted and communicated on the network. At the same time, information security of digital images is particularly important.

Information hiding technology as a common data security technology can achieve data protection by hiding the valuable data to other data information. Since image data has become an important means of information transmission, a hidden image based on the digital technology has increasingly become a research hotspot. Many literatures discussed the hidden problem of digital image $[1,2]$.

In recent years, chaotic encryption of digital images has become a hot topic. Due to the sensitivity of the initial value by chaotic system and randomness of chaotic sequence generated by the chaotic system, digital image encryption can greatly improve the security of digital image information.

So far, the spatial domain methods and transform domain embedding methods are the two main types of resolutions for image embedded technologies. By changing the spatial gray values of some pixels and the parameter values in transformable domain, these two methods have the advantages for embedding image information. The algorithms by spatial embedded are simple and effective, and can store huge information with good resistance to geometric distortion. Although transformable domain embedded image technology is of better robustness and strong anti-attack capability, the algorithm is more complex which has a small amount of 
hiding information.

Gui put forward the idea of iterative learning identification algorithm, which can hide an image or a group of images into another image using iterative mixture. Iterative mixing parameters can also be used as a key in hidden images. The method was simple and applicable, but the algorithm would be further improved in the aspects of anti-aggression and robustness [3].

Meanwhile, Yi developed an open image encryption algorithm based on chaotic sequences [4], which imported chaos theory into hidden image encryption algorithm. A digital image encryption algorithm based on spatial domain was proposed through improving the existing embedding image. However, there are a lot of studies about chaos mapping in digital image encryption which needed to be improved.

Ji derived a multi-digital image watermarking algorithm based on chaotic sequence [5]. The algorithm generated multiple one-dimensional real-valued chaotic sequences depending on different initial values which effectively solve the structural problems of multiple digital image watermark signal.

Liao proposed a three-dimensional affine transformation invariant model-the focus of the intersection distance ratio (RBC) to further enhance the confidentiality.

Yang combined wavelet transform, and proposed a digital image hiding algorithm based on discrete wavelet transform (DWT) and iterative hybrid technology [6].

Recently, Shankar and others researchers makes changes to GS (stable matching) algorithm by using of the weak stability expressed in watermark extraction systems. Due to add the iterative ideas, Fourier amplitude and phase domain templates are not constrained during iterative procedures which limit the constituents of spectrum. The algorithm achieves a blind detection phase key type of Fourier CGH digital watermarking mainly using iterative phase templates.

Therefore, considerable efforts have been devoted to the analysis of encryption and decryption with digital image. Digital image embedding and extracting technologies have made great progress at home and abroad. There are many problems to be solved, mainly in the following areas: (1) in order to measure the performance of the algorithm, embedded image system must have robustness and invisibility which are difficult to be satisfied at the same time. How to achieve a balance between these two is a research hot issue. (2) Meaningful images embedded in most images which contains copyright information such as: text, sound, images or video, usually has weak confidentiality. Therefore taking some encryption measures to better protect image copyright information, is another hot issue of digital image hiding research.

Iterative learning identification method is suitable for repeated systems. The differences between conventional time-invariant systems and time-varying systems is that iterative learning identification can be used in varying system identification and can be used to estimate the dynamic characteristics of the variable parameters. The zero identification error is achieved over the entire interval, if the learning gain meets certain convergence conditions. Sun and other scholars used iterative learning identification method to solve the problem of finite length chaotic communication of information signals $[7,8]$.

Digital images are of finite length, hidden in the chaotic system parameters of digital images, can be seen as the time varying parameters to be identified. Thus, we could apply iterative learning identification method for extracting multiple hybrid digital images for multiple embedded hybrid digital images.

This paper studies the hiding and recovery issues about the digital image by iterative learning methods. A digital image is of finite length, digital image parameters hidden in a chaotic system, can be seen as a finite length when varying parameters are identified. Therefore, we can apply iterative learning identification algorithm to recover the hidden image. The 
proposed method in this paper is finally made to test the ability for resisting JPEG compression, shear, noise, and median filtering and other geometric attacks.

The remainder of this paper is organized into four sections. Section 2 introduces the concept of mixing multiple images encryption based on a single image. Complex system with iterative learning algorithm is proposed in section 3. Section 4 substantiates the theoretical analysis with computer simulation results. Finally, Section 5 concludes this paper with final remarks.

\section{Mixture of the Digital Image}

If the images $\mathrm{F}$, and $\mathrm{G}$ are the digital images, the mixed image may be expressed as:

$$
S=\alpha F+(1-\alpha) G
$$

where constant $\alpha$ is a mixing parameter, $\mathrm{F}$ is the carrier image and $\mathrm{G}$ is the hidden image. The example of the two images blending is shown in Figure 1:

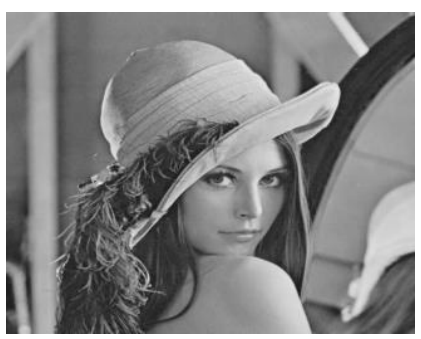

(a) carrier image

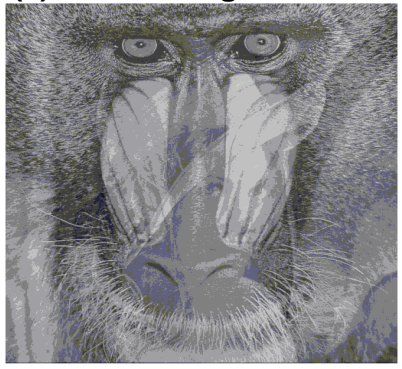

(c) $\alpha=0.3$ mixing image

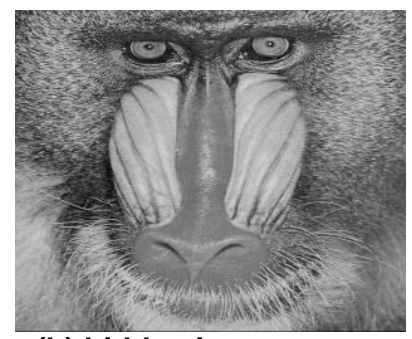

(b) hidden image

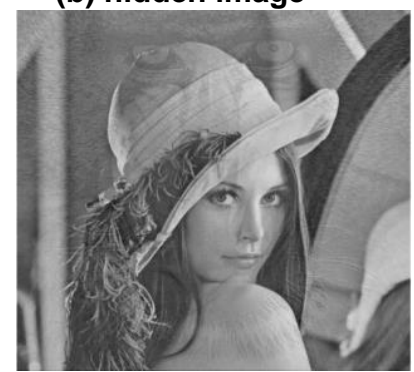

(d) $\alpha=0.9$ mixing image

Figure 1. Image Blending

The equation (1) shows that, when mixed parameter approaches 1, the mixed image $S$ is much more similar with the carrier image $\mathrm{F}$, but when mixed parameter approaches 0 , the hidden image can be easily seen to us. Therefore, choosing appropriate parameters for image blending is very important for visibility of the hidden image. Hidden image restoration method is expressed as:

$$
G=\frac{S-\alpha F}{1-\alpha}
$$

\section{Chaotic Encryption and Hiding of the Digital Image}

In order to enhance the confidentiality of the image, we introduce logistic chaotic mapping equation:

$$
x(t+1)=\mu x(t)(1-x(t)), x(t) \in[0,1]
$$


When $3.5699456 \cdots \leq \mu \leq 4$, logistic mapping showed a chaotic state. The sequence generated by the logistic mapping with given parameters $\mathrm{x}(0)$ and $\mu$ is unrelated with others.

Here we set the initial image $\theta(t)$ In order to realize the chaos encryption of hidden image, So the equation (3) can be written as follows:

$$
x(t+1)=(\lambda+\theta(t)) x(t)(1-x(t))
$$

$\{x(t), t=1,2,3, \cdots\}$ is said to be the chaotic sequence, which is the image after encryption. Flow chart with image encryption and embedded are shown in Figure 2.

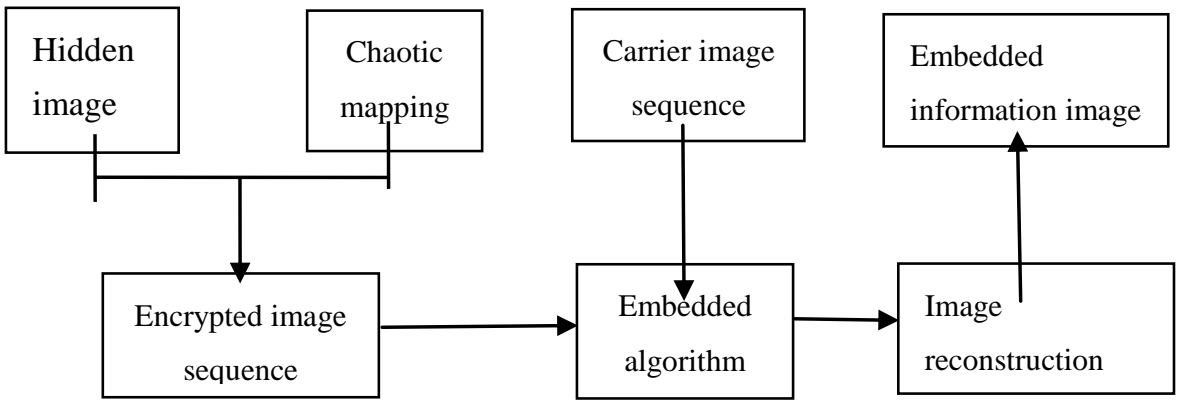

Figure 2. Encryption and Embedding of the Hidden Image

\subsection{Chaotic Encryption and Multiple Mixed with Single Image}

According to formula (1), firstly, encrypted image sequence $x(t)$ and the hidden image $F$ sequence $\mathrm{w}(\mathrm{t})$ are mixed to obtain an image with the blending parameter : $S_{1}=\alpha_{1} w(t)+\left(1-\alpha_{1}\right) x(t)$, secondly, mixing the blended image with carrier image, we get $S_{2}=\alpha_{2} w(t)+\left(1-\alpha_{2}\right) S_{1}$ then,mixing in turn, finally we obtained $S_{n}=\alpha_{n} w(t)+\left(1-\alpha_{n}\right) S_{n-1}, \quad S_{n}$ is the results of image $\mathrm{F}$ and $\mathrm{G}$ blended.

After finishing the blending procedure, the mixed image equation can be given as follows [9]:

$$
S_{n}=\left(1-\beta_{n} \beta_{n-1} \ldots \beta_{2} \beta_{1}\right) F+\beta_{n} \beta_{n-1} \ldots \beta_{2} \beta_{1} G^{\prime}
$$

where $\beta_{i}=1-\alpha_{i}, i=1,2, \ldots, n$

Using logistic mapping to generate these iteration parameters $\mu$ and $\alpha$, the parameter would expressed as:

$$
\alpha_{i+1}=\mu^{\prime} \alpha_{i}\left(1-\alpha_{i}\right)
$$

The chaotic sequence $\left\{\alpha_{i}\right\}$ is regarded as iterative sequence. The generated sequence with strong randomness can further enhance the security of the hidden image [10][11].

Single image mixing algorithm can hide a digital image into a secret image, the attacker is likely to recover the secret image when the hidden information is intercepted occasionally. So the security of the hidden systems is totally dependent on that an image carrier is vulnerable. To solve this problem, we promote the idea of mixing digital images using several mixing parameters and multiple carrier images to hide an image, which greatly enhance the image security [12].

\subsection{Chaotic Encryption and Multiple Mixed with Multiple Image}


Similarly, according to the formula (3), the hidden image is denoted as: $\theta(\mathrm{t})$, encrypted image with logistic Chaotic G'. We obtain the Relationship between hidden image and carrier images.

$$
\left\{\begin{array}{c}
S_{1}(t)=\alpha_{1} F_{1}(t)+\left(1-\alpha_{1}\right) x(t) \\
S_{2}(t)=\alpha_{2} F_{2}(t)+\left(1-\alpha_{2}\right) S_{1}(t) \\
\quad \vdots \\
S_{n}(t)=\alpha_{n} F_{n}(t)+\left(1-\alpha_{n}\right) S_{n-1}(t)
\end{array}\right.
$$

That is :

$$
\begin{aligned}
S_{n}= & \alpha_{n} F_{n}+\beta_{n} \alpha_{n-1} F_{n-1}+\cdots+\beta_{n} \beta_{n-1} \cdots \beta_{n-i} \alpha_{n-i} F_{n-i} \\
& +\cdots+\beta_{n} \beta_{n-1} \cdots \beta_{2} \beta_{1} G^{\prime}
\end{aligned}
$$

where $\beta_{i}=1-\alpha_{i}, i=1,2, \cdots, n$, according to the initial value of parameters $\mu^{\prime}$ and $\alpha_{1}$, the sequence are generated with $\alpha_{i+1}=\mu^{\prime} \alpha_{i}\left(1-\alpha_{i}\right) \cdot\left\{\alpha_{i} \mid 0<\alpha_{i}<1, i=1,2, \cdots n\right\}$. Note that the selected initial value of parameters are different from the one generated by the watermark $\mu(t)$ and $x(1)$.

\section{Iterative Learning Identification Method with Single Hidden Image}

We will attribute the recovery of hidden images to iterative learning identification problem. After encrypting the original image $\mathrm{G}$ by chaotic system, hiding it into the carrier image $\mathrm{F}$, the recovery of the original image can be reconstructed by applying iterative learning algorithm. Figure 3 shows the flow chart of restoration.

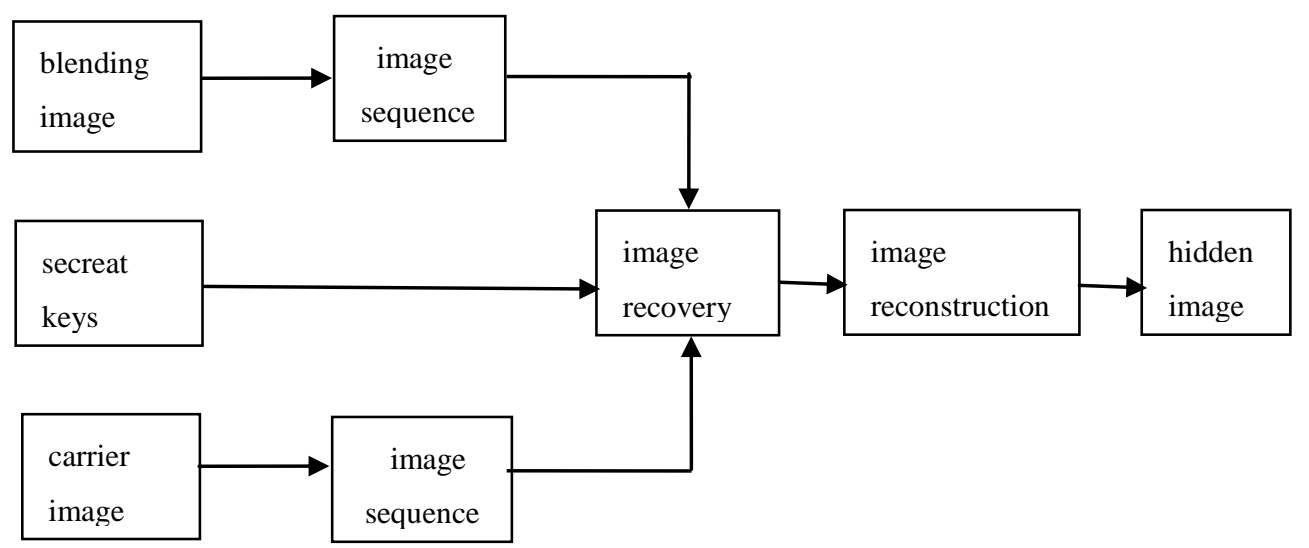

Figure 3. Recovery of the Hidden Image

Considering the following nonlinear discrete-time systems:

$$
\left\{\begin{array}{l}
x(t+1)=f(x(t), \theta(t), t) \\
y(t)=g(x(t), t)
\end{array}\right.
$$

$t \in\{0,1, \cdots, N\}, \quad x(t) \in R^{n}, \theta(t) \in R^{1}, \quad y(t) \in R^{1}, f, g \quad$ are Matrix functions. The true 
value of the parameter $\theta^{*}(t)$ is substituted into (8)

$$
\left\{\begin{array}{l}
x^{*}(t+1)=f\left(x^{*}(t), \theta^{*}(t), t\right) \\
y^{*}(t)=g\left(x^{*}(t), t\right)
\end{array}\right.
$$

iterative learning identification system used for estimation is given as follows:

$$
\left\{\begin{array}{l}
x_{k}(t+1)=f\left(x_{k}(t), \theta_{k}(t), t\right) \\
y_{k}(t)=g\left(x_{k}(t), t\right)
\end{array}\right.
$$

$k$ is the iterative number and more importantly, the initial value of each iteration is the same. Supporting that $f$ is the partial derivative of $x$ and $\theta$, and $g$ is the one of $x$.

$$
\begin{gathered}
C_{k}(t)=\left.\frac{\partial g\left(x_{k}(t), t\right)}{\partial x_{k}(t)}\right|_{x_{k}(t)=\xi_{k}(t)} \quad \xi_{k}(t)=\left(1-\sigma_{1}\right) x^{*}(t)+\sigma_{1} x_{k}(t) \quad 0<\sigma_{1}<1 \\
A_{k}(t)=\left.\frac{\partial f\left(x_{k}(t), \theta_{k}(t), t\right)}{\partial x_{k}(t)}\right|_{x_{k}(t)=\zeta_{k}(t)} \quad \zeta_{k}(t)=\left(1-\sigma_{2}\right) x^{*}(t)+\sigma_{2} x_{k}(t) \quad 0<\sigma_{2}<1 \\
B_{k}(t)=\left.\frac{\partial f\left(x_{k}(t), \theta_{k}(t), t\right)}{\partial \theta_{k}(t)}\right|_{\theta_{k}(t)=\eta_{k}(t)} \quad \eta_{k}(t)=\left(1-\sigma_{3}\right) \theta^{*}(t)+\sigma_{3} \theta_{k}(t) \quad 0<\sigma_{3}<1
\end{gathered}
$$

where the border is $C_{C}, C_{A}, C_{B}$. By using the following learning law

$$
\left\{\begin{array}{c}
\theta_{k}(t)=\operatorname{sat}\left(\vartheta_{k}(t)\right) \\
\vartheta_{k+1}(t)=\operatorname{sat}\left(\vartheta_{k}(t)\right)+\gamma_{k}(t)\left[e_{k}(t+1)-e_{k}(t)\right]
\end{array}\right.
$$

$\operatorname{sat}(\cdot)$ is known as Saturation function, $\gamma_{k}(t)$ represents learning gain, and the output error is $e_{k}(t)=y^{*}(t)-y_{k}(t)$.

According to the saturation theorem : $\theta_{k}(t)=\operatorname{sat}\left(\vartheta_{k}(t)\right), \theta^{*}(t)=\operatorname{sat}\left(\theta^{*}(t)\right)$

$$
\left\|\theta^{*}(t)-\theta_{k}(t)\right\| \leq\left\|\theta^{*}(t)-\vartheta_{k}(t)\right\|
$$

Theorem1. Iterative learning system described by the system (8) and learning law (11), if

$$
\begin{gathered}
-1 \leq \rho+\frac{\mathrm{C}_{M} \cdot \mathrm{C}_{\mathrm{B}}}{C_{A}^{\lambda}-\mathrm{C}_{\mathrm{A}}}<1 \\
\left\|1-\gamma_{k}(t) C_{k}(t+1) B_{k}(t)\right\| \leq \rho<1, \quad\left\|\gamma_{k} C_{k}(t)-\gamma_{k} C_{k}(t+1) A_{k}(t)\right\|_{\lambda} \leq C_{M} \\
\text { when } k \rightarrow \infty, \theta_{k}(t) \text { converges to } \theta^{*}(t) \text { during }\{0,1, \ldots, N\} .
\end{gathered}
$$

Proof.

$$
\tilde{x}_{k}(t)=x_{d}(t)-x_{k}(t), \quad \tilde{\theta}_{k}(t)=\theta_{d}(t)-\theta_{k}(t)
$$




$$
\begin{aligned}
& x_{d}(t)-x_{k}(t) \\
= & f\left(x_{d}(t-1), \theta_{d}(t-1), t-1\right)-f\left(x_{k}(t-1), \theta_{k}(t-1), t-1\right) \\
= & \mathrm{A}_{\mathrm{k}}(\mathrm{t}-1)\left[\mathrm{x}_{\mathrm{d}}(\mathrm{t}-1)-\mathrm{x}_{\mathrm{k}}(\mathrm{t}-1)\right]+\mathrm{B}_{\mathrm{k}}(\mathrm{t}-1)\left[\theta_{\mathrm{d}}(\mathrm{t}-1)-\theta_{\mathrm{k}}(\mathrm{t}-1)\right] \\
= & \mathrm{A}_{\mathrm{k}}(\mathrm{t}-1) \tilde{\mathrm{x}}_{\mathrm{k}}(\mathrm{t}-1)+\mathrm{B}_{\mathrm{k}}(\mathrm{t}-1) \tilde{\theta}_{\mathrm{k}}(\mathrm{t}-1) \\
= & \mathrm{A}_{\mathrm{k}}(\mathrm{t}-1)\left\{\mathrm{A}_{\mathrm{k}}(\mathrm{t}-2)\left[\tilde{\mathrm{x}}_{\mathrm{k}}(\mathrm{t}-2)\right]+\mathrm{B}_{\mathrm{k}}(\mathrm{t}-2) \tilde{\theta}_{\mathrm{k}}(\mathrm{t}-2)\right\} \\
& +\mathrm{B}_{\mathrm{k}}(\mathrm{t}-1) \tilde{\theta}_{\mathrm{k}}(\mathrm{t}-1) \\
= & \mathrm{A}_{\mathrm{k}}(\mathrm{t}-1) \mathrm{A}_{\mathrm{k}}(\mathrm{t}-2) \tilde{\mathrm{x}}_{\mathrm{k}}(\mathrm{t}-2)+\mathrm{A}_{\mathrm{k}}(\mathrm{t}-1) \mathrm{B}{ }_{\mathrm{k}}(\mathrm{t}-2) \tilde{\theta}_{\mathrm{k}}(\mathrm{t}-2) \\
& +\mathrm{B}_{\mathrm{k}}(\mathrm{t}-1) \tilde{\theta}_{\mathrm{k}}(\mathrm{t}-1) \\
= & \mathrm{A}_{\mathrm{k}}(\mathrm{t}-1) \mathrm{A}_{\mathrm{k}}(\mathrm{t}-2) \mathrm{A}{ }_{\mathrm{k}}(\mathrm{t}-3) \tilde{\mathrm{x}}_{\mathrm{k}}(\mathrm{t}-3) \\
& +\mathrm{A}_{\mathrm{k}}(\mathrm{t}-1) \mathrm{A}_{\mathrm{k}}(\mathrm{t}-2) \mathrm{B}{ }_{\mathrm{k}}(\mathrm{t}-3) \tilde{\theta}_{\mathrm{k}}(\mathrm{t}-3) \\
& +\mathrm{A}_{\mathrm{k}}(\mathrm{t}-1) \mathrm{B}_{\mathrm{k}}(\mathrm{t}-2) \tilde{\theta}_{\mathrm{k}}(\mathrm{t}-2)+\mathrm{B}_{\mathrm{k}}(\mathrm{t}-1) \tilde{\theta}_{\mathrm{k}}(\mathrm{t}-1) \\
& \quad \vdots \quad \tilde{x}_{\mathrm{A}}^{\mathrm{t}-2} \mathrm{C}{ }_{\mathrm{B}} \tilde{\theta}_{\mathrm{k}}(1)+\cdots \mathrm{C}_{\mathrm{A}} \tilde{\theta}_{\mathrm{k}}(\mathrm{t}-1) \\
= & \mathrm{A}_{\mathrm{k}}(\mathrm{t}-1) \mathrm{A}_{\mathrm{k}}(\mathrm{t}-2) \ldots \mathrm{A}_{\mathrm{k}}(0) \tilde{\mathrm{x}}_{\mathrm{k}}(0) \\
& +\mathrm{A}_{\mathrm{k}}(\mathrm{t}-1) \mathrm{A}_{\mathrm{k}}(\mathrm{t}-2) \ldots \mathrm{B}_{\mathrm{k}}(0) \tilde{\theta}_{\mathrm{k}}(0) \\
& +\mathrm{A}_{\mathrm{k}}(\mathrm{t}-1) \mathrm{A}_{\mathrm{k}}(\mathrm{t}-2) \ldots \mathrm{B}_{\mathrm{k}}(1) \tilde{\theta}_{\mathrm{k}}(1)+\cdots \mathrm{B}_{\mathrm{k}}(\mathrm{t}-1) \tilde{\theta}_{\mathrm{k}}(\mathrm{t}-1)
\end{aligned}
$$

(14)

Because of the same initial value of each iteration, we can get: $\tilde{x}_{k}(0)$, taking norm of formula (14) of both sides:

$$
\left\|\tilde{\mathrm{x}}_{\mathrm{k}}(\mathrm{t})\right\| \leq \sum_{j=0}^{t-1} C_{A}^{t-j-1} C_{B}\left\|\tilde{\theta}_{\mathrm{k}}(\mathrm{j})\right\|
$$

multiplying $C_{A}^{-\lambda t}\left(C_{A}>1\right)$ on both sizes of the equation (15), getting the max value during the interval $t \in\{0,1, \ldots T\}$,

$$
\begin{aligned}
& \left\|\tilde{\mathrm{x}}_{\mathrm{k}}(\mathrm{t})\right\|_{\lambda} \\
& \leq \sup \sum_{j=0}^{t-1}\left(C_{B} C_{A}^{t-j-1} C_{A}^{-\lambda t}\left\|\tilde{\theta}_{\mathrm{k}}(\mathrm{j})\right\|\right) \\
& \leq \frac{C_{B}}{C_{A}} \sup \sum_{j=0}^{t-1}\left(C_{A}^{t-j} C_{A}^{\lambda j-\lambda t}\left\|\tilde{\theta}_{\mathrm{k}}(\mathrm{j})\right\| C_{A}^{-\lambda j}\right) \\
& \leq \frac{C_{B}}{C_{A}} \sup \sum_{j=0}^{t-1} C_{A}^{(\lambda-1)(j-t)} \sup \left\|\tilde{\theta}_{\mathrm{k}}(\mathrm{j})\right\|_{A}^{-\lambda j} \\
& \leq \\
& \leq \frac{\tilde{\theta}_{\mathrm{k}}(\mathrm{j}) \| C_{\lambda} \frac{C_{B}}{C_{A}} \sup \sum_{j=0}^{t-1} C_{A}^{(\lambda-1)(j-t)}}{C_{A}\left(C_{A}^{\lambda-1}-1\right)}\left\|\tilde{\theta}_{\mathrm{k}}(\mathrm{t})\right\|_{\lambda}
\end{aligned}
$$


From formula (11), we know:

$$
\begin{aligned}
& \tilde{\vartheta}_{k+1} \\
& =\tilde{\theta}_{k}(t)-\gamma_{k}\left(e_{k}(t+1)-e_{k}(t)\right) \\
& =\tilde{\theta}_{k}(t)-\gamma_{k}\left\{\left[g\left(x_{d}(t+1), t+1\right)-g\left(x_{k}(t+1), t+1\right)\right]\right. \\
& \left.\quad-\left[g\left(x_{d}(t), t\right)-g\left(x_{k}(t), t\right)\right]\right\}
\end{aligned}
$$

According to the differential mean value theorem

$$
\begin{aligned}
= & \tilde{\theta}_{k}(t)-\gamma_{k}\left\{C_{k}(t+1)\left[x_{d}(t+1)-x_{k}(t+1)\right]-C_{k}(t)\left[x_{d}(t)-x_{k}(t)\right]\right\} \\
= & \tilde{\theta}_{k}(t)-\gamma_{k}\left\{C_{k}(t+1)\left[f\left(x_{d}(t), \theta_{d}(t), t\right)-f\left(x_{k}(t), \theta_{k}(t), t\right)\right]\right. \\
& \left.\quad-C_{k}(t)\left[x_{d}(t)-x_{k}(t)\right]\right\} \\
= & \tilde{\theta}_{k}(t)-\gamma_{k}\left\{C_{k}(t+1)\left\{A_{k}(t)\left[x_{d}(t)-x_{k}(t)\right]+B_{k}(t)\left[\theta_{d}(t)-\theta_{k}(t)\right]\right\}\right. \\
& \left.\quad-C_{k}(t)\left[x_{d}(t)-x_{k}(t)\right]\right\} \\
= & \tilde{\theta}_{k}(t)-\gamma_{k}\left\{C_{k}(t+1)\left\{A_{k}(t) \tilde{x}_{k}(t)+B_{k}(t) \tilde{\theta}_{k}(t)\right\}-C_{k}(t) \tilde{x}_{k}(t)\right\} \\
= & \tilde{\theta}_{k}(t)-\gamma_{k} C_{k}(t+1) B_{k}(t) \tilde{\theta}_{k}(t)-\gamma_{k} C_{k}(t+1) A_{k}(t) \tilde{x}_{k}(t)+\gamma_{k} C_{k}(t) \tilde{x}_{k}(t) \\
= & {\left[1-\gamma_{k} C_{k}(t+1) B_{k}(t)\right] \tilde{\theta}_{k}(t)-\gamma_{k} C_{k}(t+1) A_{k}(t) \tilde{x}_{k}(t)+\gamma_{k} C_{k}(t) \tilde{x}_{k}(t) } \\
= & \rho \tilde{\theta}_{k}(t)-\gamma_{k} C_{k}(t+1) A_{k}(t) \tilde{x}_{k}(t)+\gamma_{k} C_{k}(t) \tilde{x}_{k}(t) \\
= & \rho \tilde{\theta}_{k}(t)+\left[\gamma_{k} C_{k}(t)-\gamma_{k} C_{k}(t+1) A_{k}(t)\right] \tilde{x}_{k}(t)
\end{aligned}
$$

If $M_{k}=\gamma_{k} C_{k}(t)-\gamma_{k} C_{k}(t+1) A_{k}(t)$

$$
\tilde{\vartheta}_{k+1}(t)=\rho \tilde{\theta}_{k}(t)+M_{k} \tilde{x}_{k}(t)
$$

Taking norm on both of (18)

$$
\left\|\tilde{\vartheta}_{k+1}(t)\right\|_{\lambda} \leq \rho\left\|\tilde{\theta}_{k}(t)\right\|_{\lambda}+M_{k}\left\|\tilde{x}_{k}(t)\right\|_{\lambda}
$$

Note that $\left\|M_{k}\right\|_{\lambda} \leq C_{M}$, and substitute the equation (16) into (19):

$$
\left\|\tilde{\vartheta}_{k+1}(t)\right\|_{\lambda} \leq \rho\left\|\tilde{\theta}_{k}(t)\right\|_{\lambda}+\frac{C_{M} \cdot \mathrm{C}_{\mathrm{B}}}{C_{A}^{\lambda}-C_{A}}\left\|\tilde{\theta}_{\mathrm{k}}(\mathrm{t})\right\|_{\lambda}
$$

By the formula (12), the above inequality (20) can be written as:

$$
\left\|\tilde{\theta}_{k+1}(t)\right\|_{\lambda} \leq \rho\left\|\tilde{\theta}_{k}(t)\right\|_{\lambda}+\frac{C_{M} \cdot \mathrm{C}_{\mathrm{B}}}{C_{A}^{\lambda}-\mathrm{C}_{\mathrm{A}}}\left\|\tilde{\theta}_{\mathrm{k}}(\mathrm{t})\right\|_{\lambda}
$$

as long as $-1 \leq \rho+\frac{\mathrm{C}_{M} \cdot \mathrm{C}_{\mathrm{B}}}{C_{A}^{\lambda}-\mathrm{C}_{\mathrm{A}}}<1, \lim _{k \rightarrow \infty}\left\|\tilde{\theta}_{k}(t)\right\|_{\lambda}=0$.

The root mean square error(RMSE) is expressed as:

$$
R M S E=\left[\frac{1}{M N} \sum_{i=1}^{M} \sum_{j=1}^{N}[F(i, j)-S(i, j)]^{2}\right]^{\frac{1}{2}}
$$

The smaller RMSE is, the much similar between carrier image and mixed image. Here, carrier image represents $\mathrm{M} * \mathrm{M}$ pixel, while mixed image is $\mathrm{N}^{*} \mathrm{~N}$ pixel.

\section{Simulation results}

In this section, the iterative learning identification algorithm verifies the effect of extracted watermark image by experiment. According to the formula (4) and (7), selecting mixed numbers $n=5$, the state of digital watermarking system can be expressed as: 


$$
\left\{\begin{array}{l}
x_{k}(t+1)=(\lambda+m(t)) x_{k}(t)\left(1-x_{k}(t)\right) \\
y_{1}(t)=\alpha_{1} w(t)+\left(1-\alpha_{1}\right) x(t) \\
y_{2}(t)=\alpha_{2} w(t)+\left(1-\alpha_{2}\right) y_{1}(t) \\
y_{3}(t)=\alpha_{3} w(t)+\left(1-\alpha_{3}\right) y_{2}(t) \\
y_{4}(t)=\alpha_{4} w(t)+\left(1-\alpha_{4}\right) y_{3}(t) \\
y(t)=y_{4}(t)
\end{array}\right.
$$

where, setting $\lambda=3.7$, the initial value of $x_{k}(0)=0.5$. The watermark image should be binary image, carrier image is grayscale image. The watermark image mixed four times by carrier image notes as $y^{*}(t)$.

According to the formula (6), making $\mu=3.65$, the initial value of $\alpha_{1}(0)=0.6$, chaotic sequence generated by the iteration under the logistic mapping notes $\left\{\alpha_{i}\right\}$, the experimental parameters are setting $\left\{\alpha_{1}, \alpha_{2}, \alpha_{3}, \alpha_{4}\right\}$ by formula (6).

The learning gain is determined based on sufficient conditions for convergence.

$$
\gamma_{k}(t)=\frac{1}{\beta_{1} \beta_{2} \beta_{3} \beta_{4} x_{k}(t)\left(1-x_{k}(t)\right)}, \quad \beta_{i}=1-\alpha_{i}, i=1,2,3,4
$$

In order to test the performance of the proposed algorithm, we define index function: $J_{k}=\sup _{t \in\{0,1, \cdots, N\}}\left|\tilde{\theta}_{k}(t)\right|$

Figure 4 shows the original watermark image on the right and carrier image on the left, Figure 5 shows us the carrier image after the watermark image has been embedded and the extracted image using iterative learning algorithm. The experimental result demonstrates the application of the above algorithm for reconstructing the watermark image.
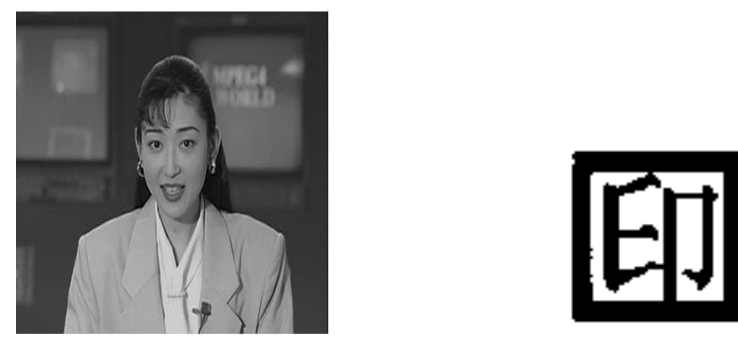

Figure 4. Original Host Image and Original Watermark Image
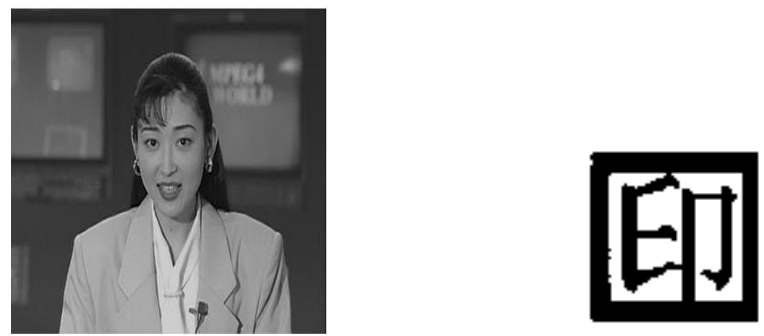

Figure 5. Watermarked Host Image and Extracted Watermark Image

The result of the anti-aggressive test algorithm is described as follows: 
(1) Resistance ability to JPEG compression
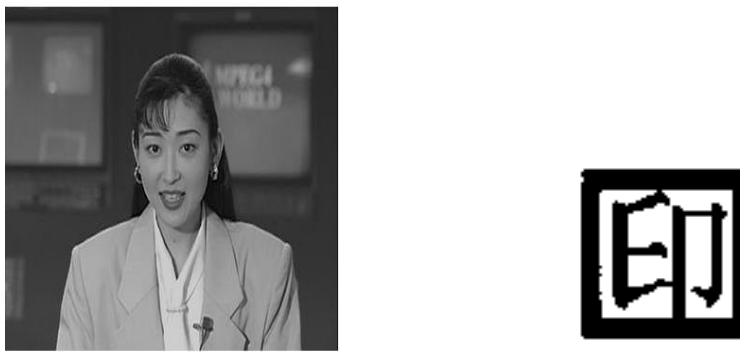

Figure 6. JPEG Compress $(Q=0.9)$ and Extracted Watermark Image
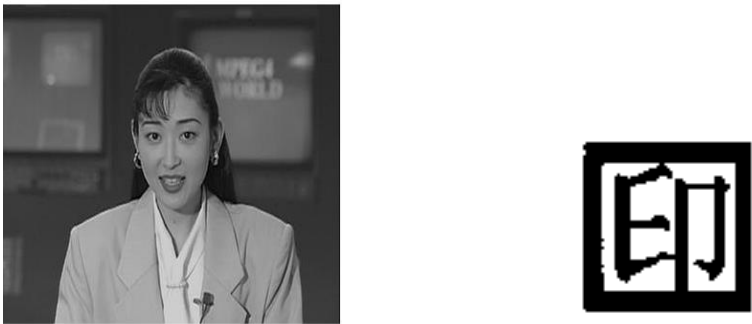

Figure 7. JPEG Compress $(Q=0.2)$ and Extracted Watermark Image

From Figure 6, Figure 7, we see the effective recovery of the watermark whatever the compressed factor is.

(2) Resistance ability to shear
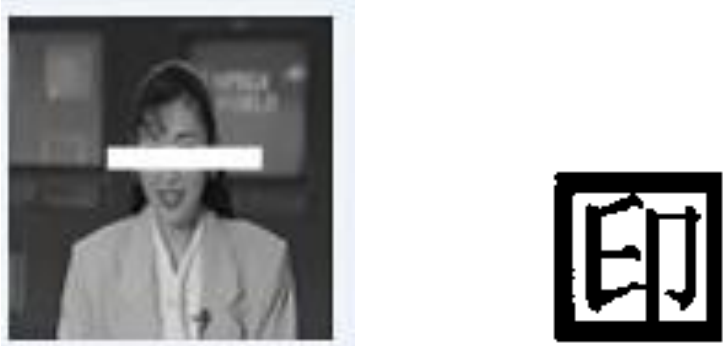

Figure 8. Cropping (Middle 12\%) and Extracted Watermark Image

(3) Resistance ability to noise
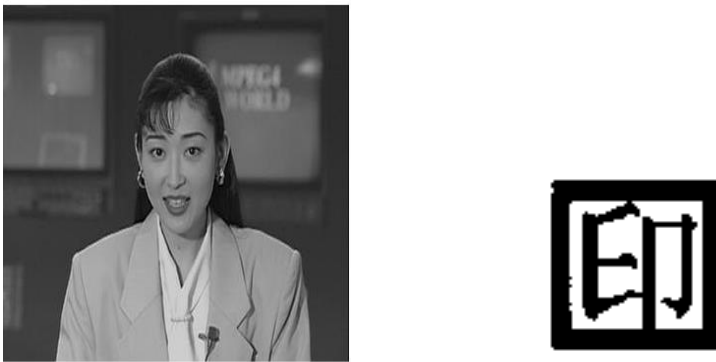

Figure 9. Adding Gaussian Noise and Extracted Watermark Image

(4) Resistance ability to median filtering 
Evaluation criteria about the digital watermarking system are judged by the two formulas: (1) normalized cross correlation which measures the similarity between the original watermark image and extracted watermark image.

$$
N C=\frac{\sum_{r=1}^{M \times M} x(t) * \hat{x}(t)}{\sum_{r=1}^{M \times M} x(t)^{2}}, x(t) \text { notes the pixels of initial watermark image, } \hat{x}(t) \text { shows the }
$$

pixels of the extracted watermark image. Pixel size of binary watermark image is $M \times M$. The larger the value of NC is, the better similarity can be shown between the original image and extracted image.

(2) Noise Ratio of peak signal(PSNR) as the objective criteria for assessing image fidelity is shown. The larger the value is, the higher the fidelity of mixed image can be reflected.

$$
P S N R=101 g \frac{N \times N \times \max \left(w^{2}(t)\right)}{\sum_{r=1}^{N \times N}(y(t)-w(t))^{2}}, \text { where } w(t) \text { is pixels of the original carrier image, }
$$

$y(t)$ demonstrates the pixels of embedded watermark image, which shows the image size $N \times N$.

Table 1 lists NC and PSNR of the embedded watermark image by attacked with JPEG compression, shearing, noises and median filtering. The experimental results verify the effectiveness of the iterative learning algorithm.

Table 1. Experimental Parameters with the Multiple Image of Multi-blending

\begin{tabular}{c|c|c|c|c}
\hline \hline Mixed parameters & $\begin{array}{l}\text { JPEG } \\
\text { compress } \\
(\mathrm{Q}=0.05)\end{array}$ & $\begin{array}{c}\text { Cropping } \\
(\text { middle } \\
12 \%)\end{array}$ & $\begin{array}{c}\text { Gaussian } \\
\text { Noise } \\
(0.01)\end{array}$ & $\begin{array}{c}\text { Mean } \\
\text { filtering } \\
(3 * 3)\end{array}$ \\
\hline PSNR & 21.4275 & 14.4589 & 20.138 & 26.568 \\
\hline NC & 0.946 & 0.798 & 0.938 & 0.937 \\
\hline
\end{tabular}

\section{Conclusion}

This paper combined chaotic mapping with iterative learning methods for researching on encryption and extracting about the watermark image problems. Under the chaotic mapping system, the watermark image can be sucessfully extracted with the iterative learning method. The experimental results showed the effectiveness and robustness of the proposed method. Future work may lie in the identification for multiple images or digital video watermarking as well as the application of iterative learning algorithms onto engineering problem solving.

\section{ACKNOWLEDGEMENTS}

This study was partly supported by National Natural Science Foundation of China (No.61272315, and No.60842009), Zhejiang Provincial Natural Science Foundation (No. Y1110342), and Zhejiang Provincial Science and Technology Department of International Cooperation Project (No.2012C24030)

\section{References}

[1] I. J. Cox and L. M. Matt, "The first 50 years of electronic watermarking”, Journal on Applied Signal Processing, vol. 2, (2002), pp. 126-132. 
[2] M. D. Swanson and M. Kobayashi, "Multimedia data embedding and watermarking technologies", Proceedings of the IEEE, vol. 86, (1998), pp. 1064-1087.

[3] Z. Gui-Cang, "Digital Image Information Hiding Technology Based on Iterative Blending", Chinese journal of computers, vol. 26, (2003), pp. 569-574.

[4] Y. Kai-Xiang, “An image encryption algorithm based on chaotic sequences”, Journal of Computer-Aided Design \& Computer Graphics, vol. 15, (2003), pp. 89-98.

[5] J. Zhen, "A multiple watermarking algorithm for digital image based on chaotic sequences", Chinese journal of computers, vol. 26, (2003), pp. 1555-1561.

[6] L. Shi-Ping, "Nonlinear N-shift masking and iterative learning identification algorithms for chaos communication", Journal of systems engineering, vol. 26, no. 3, (2011), pp. 560-570.

[7] A. Bemporad and F. Borrelli, "Model predictive control based on linear programming the explicit solution", IEEE Transaction on automatic control, vol. 47, no. 12, (2002), pp. 1974-1985.

[8] P. Tondel and T. A. Johansen, "An algorithm for multi-parametric quadratic programming and explicit MPC solutions", Automatics, vol. 39, (2003), pp. 489-497.

[9] W. Feng-Jian, "Research of Chaotic Block Cipher Algorithm Based on Logistic Map", In the proceeding of Second International Conference on Intelligent Computation Technology and Automation, (2009) July 14-17, Chongqin, China.

[10] F. Yu, "A Wavelet Digital Watermarking Algorithm Based on Chaotic Mapping", In the proceeding of Electronic Commerce and Security, (2008) September, 15-18, Kyoto, Japan.

[11] D. Tuia, "Explicit Recursive and Adaptive Filtering in Reproducing Kernel Hilbert Spaces", IEEE Transactions on Neural Networks and Learning Systems, vol. 25, (2013), pp. 1413-1419.

[12] A. Beirami, "A Framework for Investigating the Performance of Chaotic-Map Truly Random Number Generators", IEEE Transactions on Circuits and Systems II: Express Briefs, vol. 60, (2013), pp. 446-450.
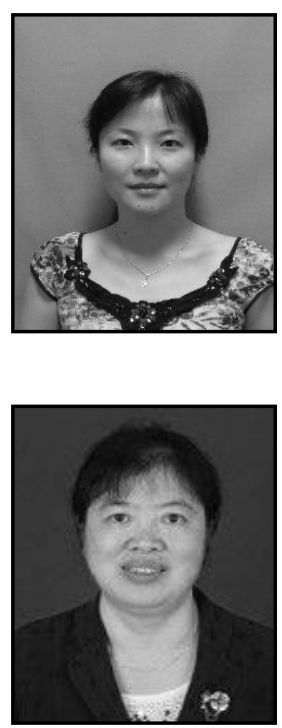

\section{Authors}

Kong Ying was born in Hangzhou, China, in 1980. She received the B.S. Degree in computer science and technology from Zhejiang Gongshang University, Hangzhou, China, in 2003. She received the M.S. Degree in computer technology from Zhejiang University in 2008. She is currently working toward Ph.D. degree in iterative learning systems at the school of Information and Technology, Zhejiang University of Technology, Hangzhou, China. Her main researches include neural network, pattern recognition, intelligent information processing.

Lu Hui-Juan is a teacher of China Jiliang University. She received B.S. Degree in industrial electrical automation from China University of Mining and Technology, Xuzhou, China and the Ph.D. Degree from China University of Mining and Technology, in 2012. 